\title{
Watching an Egg Cook with X Rays
}

\section{An x-ray scattering technique reveals how egg whites gel on a range of length and timescales.}

\section{By Erika K. Carlson}

W

hen the white of an egg cooks, the protein molecules it contains unfold and link together, forming a network that turns the viscous goop into a solidified gel. Now, Nafisa Begam of the University of Tübingen, Germany, and colleagues have experimentally constructed a detailed picture of how the proteins form this structure [1].

Understanding how an egg white transforms in the presence of heat is of interest to the food industry-because of the food's many uses in cooking and baking-as well as to researchers studying the behaviors of colloidal gels and proteins.

Researchers have studied egg white gelation at molecular scales to see how individual proteins unfold and link to their neighbors. But a full understanding of the gelation process requires observing the substance on larger, micrometer scales-something that was not possible with past methods. In their experiments, Begam and colleagues extracted the white from a store-bought chicken egg and then heated it on a lab heating stage. They probed the white using $x$-ray photon correlation spectroscopy along with ultrasmall-angle $x$-ray

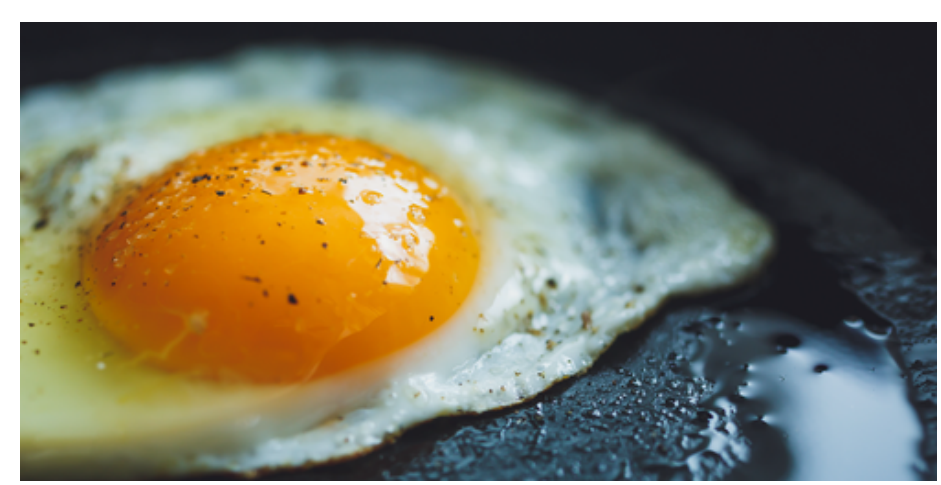

Credit: kkolosov/stock.adobe.com scattering techniques that allowed them to watch the gelation happen at the micrometer scale of the resulting network's mesh size.

The team found that the network formed in the white within a couple of minutes of the heat being applied; after that, no further structure developed. Meanwhile, the gel's dynamics showed two clear regimes: The relaxation time-the time it takes parts of the network to reach some "relaxed" state after experiencing stresses during network formation-first grew exponentially and then settled to a near-constant value. The team says that the $x$-ray scattering techniques they used may be applied to uncover the dynamics of other soft systems over a range of length scales.

Erika K. Carlson is a Corresponding Editor for Physics based in New York City.

\section{REFERENCES}

1. N. Begam et al., "Kinetics of network formation and heterogeneous dynamics of an egg white gel revealed by coherent x-ray scattering," Phys. Rev. Lett. 126, 098001 (2021). 